\title{
Abhandlungen
}

\author{
Wolfgang Streeck
}

\section{Kontinuität und Wandel im deutschen System der industriellen Beziehungen: Offene Fragen*}

\begin{abstract}
Trotz tiefgehenden wirtschaftlichen und politischen Wandels erscheint das deutsche System der industriellen Beziehungen bemerkenswert stabil. Neue Forschungsergebnisse über den Einfluss der Internationalisierung auf die Beziehungen zwischen Arbeitgeber und Arbeitnehmern in den einhundert größten deutschen Unternehmen bestätigen diesen Eindruck. Genauere Betrachtung zeigt jedoch eine wachsende Abhängigkeit des Tarifverhandlungssystems von dem System der sozialen Sicherung; Tendenzen zu einem verfestigten Dualismus im Arbeitsmarkt und im Arbeitsmarktregime; eine Einkapselung des ursprünglich universalistischen Systems industrieller Bürgerrechte in ein schrumpfendes Segment der Wirtschaft; eine zunehmende Entpolitisierung und „Privatisierung“ von Beteiligungsrechten; einen Trend zur Integration von Interessenvertretung am Arbeitsplatz in die Arbeitsorganisation; und einen Rückgang der Fähigkeit des Nationalstaats, in die private Sphäre großer Unternehmen einzugreifen.
\end{abstract}

\section{Kontinuität}

Seit den siebziger Jahren berichten die einschlägigen Untersuchungen über Struktur und Funktion des deutschen Systems der industriellen Beziehungen vor allem von einer im internationalen Vergleich erstaunlichen Kontinuität. Anderswo, etwa in den anglo-amerikanischen Ländern, aber auch in Schweden, mag es zeitweise hoch her oder gar drunter und drüber gegangen sein - dem deutschen Flächentarif, der Mitbestimmung, den gut organisierten deutschen Gewerkschaften und Arbeitgeberverbänden sowie dem konsensualen Charakter der Beziehungen zwischen Tarifparteien und Staat haben, so liest man immer wieder, und nicht ohne Grund, weder die europäische Integration noch die deutsche Wiedervereinigung wirklich etwas anhaben können:

1. Flächentarif und Tarifautonomie sind weitgehend intakt geblieben. Versuche oder auch nur Drohungen des Staates, in die Vertragsfreiheit der Tarifparteien einzugreifen, hat es, anders als etwa in den Niederlanden, nie wirklich gegeben. Der Deckungsgrad der Flächentarife mag an seinen Rändern, besonders in Ostdeutschland, zurückgegangen

* Thesen zur Diskussion auf einem Workshop des Max-Planck-Instituts für Gesellschaftsforschung, Köln, 8. und 9. Dezember 2000. Das Manuskript wurde geringfügig überarbeitet. 
sein; immer noch gehört Deutschland aber zu den Ländern, in denen die Löhne und Arbeitsbedingungen der überwiegenden Mehrzahl der Beschäftigten durch überbetrieblich ausgehandelte Tarifvereinbarungen festgelegt werden (Traxler 1996). Es trifft zu, dass Verhandlungsmaterie von der überbetrieblichen auf die betriebliche Ebene abgewandert ist; in der großen Mehrzahl der Fälle geschah dies jedoch mit Duldung oder gar formaler Ermächtigung durch die Tarifparteien, die gelernt haben, sich der Organe der betrieblichen Mitbestimmung als Vehikel einer kontrollierten Dezentralisierung zu bedienen, die das System branchenweiter Tarifverhandlungen eher stützt als untergräbt und deshalb auch als Beleg für die Anpassungsfähigkeit und damit die Kontinuität des Systems angesehen werden kann. Soweit in Deutschland überhaupt tarifvertragsfreie Zonen oder Bereiche entstanden sind, in denen geltende Tarifverträge in größerem Ausmaß von Unternehmen oder Belegschaften missachtet werden, liegen diese in Ostdeutschland. Nach Westen ausgeweitet haben sie sich bisher nicht, und solange dies so bleibt, geht es eigentlich nur darum, genügend Geduld aufzubringen, bis sich die erprobten und bewährten westlichen Regelungen auch im Osten fest etabliert haben werden.

2. Ähnliches gilt für die Mitbestimmung - auf Betriebs- wie auf Unternehmensebene: Zwar erwies sich die Mitbestimmung im Aufsichtsrat zum Leidwesen der deutschen Gewerkschaften als nicht in die EU exportierbar; entgegen manchen Befürchtungen wird sie aber auch nicht durch ein europäisches Gesellschaftsrecht ausgehebelt werden. Heute, so scheint es, hat sie sich in einem europäischen Flickenteppich konkurrierender Mitbestimmungsregime insgesamt komfortabel eingerichtet. Auch das deutsche Gesetz zur Kontrolle und Transparenz im Unternehmensbereich (KontraG) aus dem Jahre 1999 liess die Aufsichtsratsmitbestimmung unangetastet - wie überhaupt festzustellen ist, dass die 1972 und 1976 von der sozialliberalen Koalition verabschiedeten Mitbestimmungsgesetze insofern Epoche machend waren, als sie bis heute in ihrer rechtlichen Substanz unverändert geblieben sind, auch in den langen sechzehn Jahren der Ära Kohl. Dasselbe gilt für die nach wie vor starke Rolle der Gewerkschaftenin den Mitbestimmungsorganen, die der in vielen Betrieben und Unternehmen zu beobachtenden informellen Ausweitung der Beteiligung der Belegschaft an der Unternehmensführung bei gleichzeitiger Vertiefung der wirtschaftlichen Kooperation nicht nur nicht im Wege stand, sondern sie häufig geradezu ermöglicht hat. Versuche von Arbeitgebern, Betriebsräte einzuschüchtern oder gar abzuschaffen, gibt es ebenso wie gewerkschaftsfreie oder gar gewerkschaftsfeindliche betriebliche Kooperation; die Regel sind beide aber nicht. Zwar hat, wie unter anderem die Kommission Mitbestimmung (Kommission Mitbestimmung 1998) festgestellt hat, der Anteil der Beschäftigten, die durch Betriebsräte vertreten werden, in den letzten anderthalb Jahrzehnten nicht unbeträchtlich abgenommen. Neben dem sich hinziehenden Nachbau des westlichen Systems im Osten scheinen hierfür vor allem der Strukturwandel hin zu kleineren Betriebseinheiten und zum Dienstleistungssektor sowie allgemein neue betriebliche Organisationsformen verantwortlich zu sein, die der Wirklichkeit von 1972, als das geltende Betriebsverfassungsgesetz geschrieben wurde, nicht mehr entsprechen. So problematisch dies aber sein mag, so die Stabilitäts- und Kontinuitätsthese, es müsste sich durch zeitgemäße Reparaturen an den gesetzlichen Grundlagen der Betriebsverfassung beheben lassen, und eben dies hat ja die gegenwärtige, sozialdemokratisch geführte Bundesregierung auf Druck der Gewerkschaften mit der Novellierung des Betriebsverfassungsgesetzes gerade unternommen. 
3. Was die Organisationen der Sozialpartner angeht, so haben sich Ankündigungen eines bevorstehenden Zerfalls der Arbeitgeberverbände offenbar als verfrüht erwiesen (Schroeder/Ruppert 1996). Sieht man wiederum von Ostdeutschland ab, scheint sich die Organisationsquote insgesamt kaum verändert zu haben, vor allem wenn man die sogenannten OT-Verbände einbezieht, also jene ,zweiten Anzüge“, die einige Arbeitgeberverbände sich in den neunziger Jahren zugelegt haben, um Firmen die Vorteile einer Verbandsmitgliedschaft bieten zu können, ohne von ihnen dafür Unterwerfung unter den Flächentarif verlangen zu müssen. Interessant ist, dass die Möglichkeit des Übertritts in einen OT-Verband anscheinend nicht, wie manchmal befürchtet, eine Flucht aus den klassischen Arbeitgeberverbänden ausgelöst hat; vielmehr gibt es Übertritte in beide Richtungen, die sich insgesamt die Waage zu halten scheinen. Schwieriger scheint die Situation bei den Gewerkschaften, die als Folge des Strukturwandels, wohl auch des Rückgangs der Betriebsratsdeckung sowie niedriger Beschäftigung und eines dramatischen Einbruchs der Mitgliedschaft im Osten heute in Gesamtdeutschland nicht mehr Mitglieder haben als in Westdeutschland vor der Wiedervereinigung, bei weiterhin fallender Tendenz. Konsequenz ist unter anderem eine tiefe gewerkschaftliche Finanzkrise, verbunden mit einem Personalüberhang, der eine Erneuerung und Verjüngung des Funktionärskörpers außerordentlich erschwert. Ob und wie sich die Mitgliederverluste umkehren lassen, ist alles andere als klar; ein weithin als vielversprechend empfundener Weg sind Fusionen, mit denen die Organisationsgrenzen an neue Branchenstrukturen angepasst, Reibungsverluste bei der Mitgliederwerbung vermieden und die Kosten der Werbung und Betreuung von Mitgliedern gesenkt werden sollen. Weitere gewerkschaftliche Hoffnungen richten sich auf die Reform der Betriebsverfassung, die wie nach 1972 die institutionelle Präsenz der Gewerkschaften im Betrieb und damit ihre Möglichkeiten zur Werbung von Mitgliedern verbessern soll.

4. An der staatlichen Politik gegenüber den Tarifparteien schließlich, und insbesondere an dem Interesse des Staates an dreiseitigen Absprachen unter Einbeziehung der Gewerkschaften, scheint sich über die Jahre ebenfalls wenig geändert zu haben. Noch in der Spätphase der Kohl-Regierung gab es eine sogenannte „Kanzlerrunde“, die 1996 beinah in ein formelles „Bündnis für Arbeit“ eingemündet wäre. Versuche der Regierung, im Rückblick eher geringfügig erscheinende Reformen des Sozialstaats und des Arbeitsmarktregimes ohne Beteiligung der Gewerkschaften und gegen diese durchzusetzen, trugen wahrscheinlich zur Wahlniederlage der CDU/CSU 1998 bei. Die SPD übernahm das „Bündnis“-Projekt, zunächst um im Wahlkampf Fragen nach der von ihr beabsichtigten Arbeitsmarkt- und Beschäftigungspolitik ausweichen zu können. Die nach dem Regierungswechsel begonnenen dreiseitigen Gespräche haben bis heute kaum greifbare Ergebnisse hervorgebracht; insbesondere aus der Perspektive derjenigen innerhalb und außerhalb der Bundesregierung, die sich von ihnen institutionelle Reformen des Arbeitsmarktes versprochen hatten, haben sie sich als Fehlschlag erwiesen (Streeck 2001a).

Auch die Gewerkschaften haben sich über ausbleibende „Ergebnisse“ der Bündnisgespräche beklagt. Aber wenn überhaupt, so belegt dies wahrscheinlich lediglich das Fortbestehen eines nach wie vor hohen politischen Anspruchsniveaus. Unerwähnt bleibt, dass die Wiederbelebung des Korporatismus in den neunziger Jahren den Gewerkschaften in Deutschland, anders als in Italien oder den Niederlanden, so gut wie keine Opfer abverlangt hat - etwa in Bezug auf die Flexibilisierung des Arbeitsmarkts, die Struktur des Lohnfindungssystems oder die Praxis der Arbeitsmarktpolitik. Im Gegenteil räumt sie ihnen eine äußerst wirksame Vetopo- 
sition ein, von der sie in den ersten drei Jahren der Regierung Schröder mit wachsendem Selbstbewusstsein Gebrauch gemacht haben.

\section{Kontinuität im Wandel}

Betrachtet man die ersten sich abzeichnenden Ergebnisse der am MPIfG durchgeführten Arbeiten zum Einfluss der Internationalisierung auf die industriellen Beziehungen in den einhundert größten deutschen Unternehmen, so lassen sich auch diese zunächst im Sinne einer Stabilitäts- und Kontinuitätsthese interpretieren ${ }^{1}$. Generell war, wie schon der Bericht der Kommission Mitbestimmung dargelegt hat, die Internationalisierung sowohl der Absatzmärkte als auch der Produktionsstrukturen für deutsche Unternehmen trotz der Besonderheiten ihrer industriellen Beziehungen nicht schwierig und auch ohne einen Gestaltwandel in den Arbeitsbeziehungen wirtschaftlich erfolgreich zu bewältigen, einschließlich eines in den neunziger Jahren zeitweise dramatischen inländischen Beschäftigungsabbaus. Auch im Einzelnen finden sich bemerkenswerte Beispiele für hohe Kontinuität unter Anpassungsdruck:

1. So erscheinen die in fast allen untersuchten Unternehmen mehr oder weniger verbreiteten sogenannten Standortvereinbarungen bei näherem Hinsehen als mit dem überkommenen institutionellen Rahmen der deutschen Arbeitsbeziehungen keineswegs unvereinbar, und zwar nicht nur, weil die jeweils geltenden tarifvertraglichen Regelungen nur selten verletzt und in der Regel nahezu peinlich genau eingehalten werden - schon weil die zuständigen Gewerkschaften an der Aushandlung von Standortvereinbarungen oft direkt oder indirekt beteiligt sind. Vor allem erweist sich, was zunächst als deutsche Version des amerikanischen , concession bargaining “ der achtziger und frühen neunziger Jahre hätte erscheinen können, spätestens dann eher als Verlängerung und informelle Ausweitung der deutschen Tradition ausgehandelter Kooperation, wenn die beteiligten Unternehmensleitungen sich auf Zusagen über das künftige Beschäftigungsniveau sowie über am „Standort“ geplante Investitionen einlassen. Zwar sind diese zumeist nicht rechtsverbindlich; in der Praxis aber werden sie von beiden Seiten in erstaunlich hohem Maße als bindend empfunden. Dementsprechend hat es auch dann, wenn multinationale Unternehmen einen regelrechten Wettbewerb zwischen Produktionsstandorten in verschiedenen Ländern veranstalteten, bei dem örtliche Belegschaften mit Vorschlägen zur Kostensenkung oder zur Steigerung ihrer Arbeitsproduktivität um zentral zugeteilte Investitionsmittel konkurrieren müssen, erstaunlich selten offene Konflikte zwischen Management und Betriebsräten oder Gewerkschaften gegeben. Es trifft zu, dass viele der in den neunziger Jahren geschlossenen Standortvereinbarungen ihre Bewährungsprobe noch vor sich haben. Auch könnten weitere Vereinbarungen wegen des kleiner gewordenen übertariflichen Manövrierspielraums es immer schwerer finden, Konflikten mit dem Flächentarif aus dem Weg zu gehen. Andererseits gibt es aber Anzeichen dafür, dass die Tarifparteien zunehmend bereit sind, in die sektoralen Tarifverträge erweiterte Regelungsspielräume

1 Der folgende Abschnitt fasst aus der Sicht des Autors einige ausgewählte und vorläufige Ergebnisse eines Forschungsprojekts des Max-Planck-Instituts für Gesellschaftsforschung (MPIfG) über den Einfluss der Internationalisierung auf das deutsche System der industriellen Beziehungen zusammen, an dem neben dem Autor Anke Hassel, Martin Höpner, Antje Kurdelbusch, Britta Rehder und Rainer Zugehör beteiligt sind. Genaue Informationen über das Projekt und die bisher aus ihm hervorgegangenen Veröffentlichungen finden sich auf der Homepage des MPIfG (http://www.mpi-fg-koeln.mpg.de). 
einzubauen, die das wachsende Interesse der Betriebsparteien an informellen Vereinbarungen über Produktivität, Investitionen und Beschäftigung berücksichtigen. Damit spricht vieles dafür, dass Standortvereinbarungen zu einem zusätzlichen Element im Instrumentarium der deutschen Sozialpartnerschaft auf betrieblicher und möglicherweise sogar tariflicher Ebene werden könnten oder schon geworden sind.

2. Ähnliches gilt für die sich immer weiter ausbreitende Praxis kontingenter Entlohnung, also die Kopplung eines Teils der Arbeitnehmereinkommen an die individuelle Leistung oder den Unternehmenserfolg. Die überwiegende Mehrzahl der deutschen Großunternehmen experimentiert heute mit unternehmensspezifischen Entlohnungsregimen, die entweder der Flexibilisierung der Kosten (ertragsabhängige Entlohnung) oder der Erhöhung der Produktivität durch verbesserte Arbeitsanreize (leistungsabhängige Entlohnung) dienen sollen. Zwar bleibt deren tatsächliche Einführung bis heute zumeist auf den außer- und übertariflichen Bereich beschränkt; an der Absicht, sie in Zukunft möglichst tief in die derzeitige Regelungssphäre der Tarifverträge hinein auszuweiten, kann jedoch kein Zweifel bestehen. Die Tradition konsensualer Beteiligung der Belegschaftsvertretung bleibt davon allerdings unverändert unberührt. In der überwiegenden Mehrzahl der Großunternehmen ist der Betriebsrat an den Überlegungen der Geschäftsleitung zur Ausweitung kontingenter Entlohnungsformen beteiligt, und Austritte aus dem Arbeitgeberverband und damit aus dem Flächentarif, um kontingente Entlohnung schneller und umfänglicher durchsetzen zu können, scheint es bisher nicht gegeben zu haben. Obwohl Betriebsräte und Unternehmensleitungen über die Ausgestaltung eines wünschenswerten Lohnregimes häufig anderer Ansicht sind, entspricht die Wirklichkeit eher dem Bild eines gemeinsamen Experimentierens als dem eines grundsätzlichen Konflikts, zumal durchaus auch zwischen den Unternehmensleitungen die Ansichten darüber auseinandergehen, welcher Teil der Gesamtentlohnung im optimalen Fall kontingent werden bzw. konstant bleiben sollte. Ebenso wie bei den Standortvereinbarungen gibt es im Übrigen auch hier weit mehr Anzeichen für eine Anpassung der Flächentarife an sich verändernde betriebliche Regelwerke als für einen Verdrängungswettbewerb zwischen diesen und dem System der Flächentarife.

3. Auch der zunehmende Druck des Kapitalmarkts scheint, anders als weithin vermutet, die industriellen Beziehungen deutscher Großunternehmen nicht zu destabilisieren. Ebensowenig wie bei den Produktmärkten bedarf es anscheinend bei den Kapitalmärkten für eine erfolgreiche Anpassung der Unternehmen an veränderte wirtschaftliche Bedingungen eines Ausschlusses der Vertreter der Belegschaften von der Unternehmensführung. Insbesondere die Orientierung der Unternehmen am sogenannten , Shareholder Value “ scheint am Widerstand von Betriebsräten und Gewerkschaften weder zu scheitern, noch scheint sie deren institutionelle Schwächung vorauszusetzen oder zur Folge zu haben. Die von internationalen Anlegern verlangte verbesserte Transparenz der Rechnungslegung von Großunternehmen kommt durchaus auch den Gewerkschaften und Betriebsräten zugute, die nun weit besser als in der Vergangenheit über die wirtschaftliche Leistungsfähigkeit des Unternehmens informiert sind.Aktienoptionen für das Management sind vor allem dann für die Belegschaft akzeptabel, wenn zugleich die Beteiligung der langfristig beschäftigten Belegschaftsmitglieder am Unternehmenskapital verbessert wird. Da auch Belegschaftsaktien als eine Art kontingenter Entlohnung angesehen werden können, können weder Aktionäre noch Management gegen sie grundsätzliche Einwände haben; insofern als Belegschaftsaktien den Anteil langfristig interessierter Anleger an den 
Eigentümern des Unternehmens vergrößern, liegt ihre möglichst breite Einführung übrigens im direkten Interesse des Managements. Hinzu kommt, dass Belegschaftsaktien geeignet sind, den Widerstand der Arbeitnehmervertreter gegen die Einführung von Renditezielen, die in der Regel mit einer Umverteilung des Unternehmensergebnisses zugunsten der Aktionäre verbunden ist, zu verringern. Grundsätzlich gilt ferner, dass Arbeitnehmervertreter das Interesse der Unternehmensleitungen an einem hohen Aktienkurs als Mittel zur Verhinderung einer immer möglicher gewordenen feindlichen Übernahme teilen; so finden sich zunehmend Fälle, in denen die Vertreter der Arbeitnehmer das Management geradezu drängen, auch schmerzhafte Maßnahmen zur Kurspflege nicht weiter aufzuschieben. Zu diesen kann auch der Verkaufweniger ertragreicher Unternehmensteile gehören, wenn und soweit dafür gesorgt wird, dass die Belastung der betroffenen Belegschaftsteile in erträglichen Grenzen bleibt. Selbst gegen feindliche Übernahmen scheint es kaum mehr prinzipielle Widerstände zu geben; die Zeiten, in denen Gewerkschaften und Betriebsräte die „Deutschland AG“ mobilisierten, um das Entstehen eines „Marktes für Unternehmenskontrolle“ zu verhindern, scheinen endgültig vorbei. An die Stelle politischer Proteste dürften zunehmend, wie im Fall Mannesmann, industriepolitische Forderungen treten - etwa bezogen auf die Art der anschließenden Verselbständigung einzelner Unternehmensteile -, für deren Durchsetzung in Deutschland grundsätzlich genug politischer und wirtschaftlicher Handlungsspielraum zu bestehen scheint.

4. Schließlich scheint auch der kapitalmarktinduzierte Strukturwandel der international tätigen Großunternehmen einer kooperativen und kooperationsfördernden Vertretung der Interessen der Belegschaften im Rahmen der bestehenden Institutionen der industriellen Beziehungen nicht grundsätzlich im Wege zu stehen. Erste Forschungsergebnisse zeigen, dass deutsche Konglomerate, deren Anteile am Kapitalmarkt gehandelt werden, eine Strategie der Entdiversifizierung verfolgen, um ihren Aktienkurs zu erhöhen und sich so gegen feindliche Übernahmen zu schützen. Dies muß jedoch nicht gegen den Widerstand der Belegschaften bzw. ihrer gewählten Vertreter und kann im Gegenteil in der Mehrzahl der Fälle mit deren Unterstützung geschehen, wobei ein konsensuales „CoManagement“ des Unternehmensumbaus im Rahmen einer die Belegschaften einschließenden „Entdiversifizierungskoalition“ der Tiefe und Geschwindigkeit des Strukturwandels sogar förderlich zu sein scheint. Auch spricht in den bis jetzt vorliegenden Forschungsergebnissen nichts dafür, dass deutsche Unternehmen durch die Globalisierung der Finanzmärkte gezwungen wären, jene Art von langfristig rentablen Investitionen zu vernachlässigen, an denen insbesondere den gewählten Arbeitnehmervertretern liegt und deren Kultivierung als eine der wichtigsten traditionellen Geschäftsgrundlagen der deutschen Sozialpartnerschaft gelten kann - also vor allem Investitionen in Forschung und Entwicklung sowie die Qualifikationen der Belegschaft. Tatsächlich scheint ein ausreichend großes Segment des globalen Kapitalmarktes Anlagen in Unternehmen zu schätzen, die - aus welchen Gründen auch immer - dazu disponiert sind, ihre Gewinnperspektiven eher langfristig zu definieren. Dafür, dass Kapitalmärkte und Analysten deutsche Großunternehmen wegen der Mitbestimmung mit einem Abschlag bei ihren Aktienkursen bestrafen, gibt es im übrigen keinerlei Anhaltspunkte.

Freilich bleibt offen, wie derartige Beobachtungen zu werten sind. Folgt man Autoren wie Thelen (1999) oder Soskice (1999), so fallen auch die von ihnen zugestandenen Krisenerscheinungen - die abnehmende Tarifdeckung, die wachsende mitbestimmungsfreie Zone 
und der mittlerweile beträchtlich gesunkene gewerkschaftliche Organisationsgrad - gegenüber dem alles überragenden und von der empirischen Forschung immer wieder bestätigten Sachverhalt kaum ins Gewicht, dass die deutschen Arbeitgeber das Prinzip kooperativer, Gewerkschaften und Betriebsräte einbeziehender Arbeitsbeziehungen bislang, trotz gelegentlicher, zum Geschäft gehörender Androhung des Gegenteils, nicht aufgekündigt haben. Wenn es, wie die an Swenson (1997) und andere anschließende revisionistische Literatur behauptet, in der politischen Ökonomie nicht in erster Linie auf die Solidarität der Arbeitnehmer oder die Macht sozialistischer Parteien und Bewegungen ankommt, sondern auf die Strategiewahl der Arbeitgeber, dann verläuft die entscheidende Konfliktlinie ohnehin nicht zwischen den Klassen oder dem, was von ihnen übriggeblieben ist, sondern, in Deutschland, zwischen den Herren Hundt und Henkel. Und wieviel Geschrei der letztere in seiner nunmehr abgelaufenen Amtszeit auch veranstaltet haben mag: dass nicht er am Ende gewonnen hat, sondern seine biederen sozialpartnerschaftlichen Gegenspieler, daran kann ebensowenig Zweifel bestehen wie daran, dass dieser Ausgang der einzig möglich war: aus der Perspektive einer funktionalistischen Theorie der politischen Voraussetzungen der spezifisch deutschen Produktionsweise ebenso wie aus der einer historisch-institutionalistischen Theorie der „Pfadgebundenheit“ auch von Unternehmensentscheidungen, und allemal aus der Perspektive beider zusammen.

Wem Prognosen dieser Art zu schön vorkommen, um wahr sein zu können, für den gibt es auch pessimistischere und in Bezug auf den Ausgang des Geschehens weniger deterministische Versionen der Kontinuitätsthese - Versionen, die empirische Sachverhalte wie das nun doch schon länger andauernde Schrumpfen der Reichweite des deutschen Systems der industriellen Beziehungen ernster nehmen. Eine solche Sichtweise würde zumindest als Möglichkeit anerkennen, dass graduelle Veränderungen innerhalb eines sozialen Systems dieses an Grenzen führen können, die a priori schwer zu bestimmen sein mögen, jenseits derer aber jedenfalls ein neues Spiel in einem neuen System beginnt. Optimismus bestünde dann darin, dass man es für möglich hält, dass die an die Grenzen des Systems gehenden Veränderungen angehalten und rückgängig gemacht werden können - dass also, im Fall des deutschen Systems der industriellen Beziehungen, die derzeitige Phase einer existenz- bzw. identitätsbedrohenden Schrumpfung in womöglich zyklischem Wechsel von einer neuen Wachstumsphase abgelöst werden könnte, als Folge organisatorischer Unterstützung durch eine sozialdemokratische Regierung, eines neuen Betriebsverfassungsgesetzes, der Gründung von Ver.di, einer Wiederholung von 1969 oder wessen auch immer. Allerdings scheint es, dass die Suche nach Kandidaten für Faktoren oder Ereignisse, die als Verursacher eines Trendwechsels in Frage kommen könnten, den Optimismus, um dessentwillen und mit dem sie begonnen wurde, beeinträchtigen kann.

\section{$3 \quad$ Wandel}

Im Folgenden geht es mir nicht darum, ein weiteres Mal die Evidenz für und wider die Stabilitäts- und Kontinuitätsthese gegeneinander abzuwägen. Statt dessen möchte ich darüber spekulieren, was denn das Neue sein könnte, wenn es, allem ökonomisch-funktionalistischen und soziologisch-pfadkonservativen Determinismus zum Trotz, tatsächlich unterwegs wäre. Lassen sich heute, unter Einschluss auch derjenigen tatsächlichen Entwicklungen, die gemeinhin als Belege für die Stabilität des Systems ins Feld geführt werden, Ordnungsmuster 
und Problemkataloge identifizieren, die die Zukunft der Arbeitsbeziehungen in Deutschland auf eine Weise bestimmen könnten, die es erlauben und sogar erforderlich machen würden, von einer in relevanter Hinsichtdiskontinuierlichen Entwicklung zu sprechen? Dabei werde ich nicht den Anspruch stellen, dass die von mir zu beschreibenden Tendenzen sämtlich zueinander ,passen“; das ist schon deshalb nicht erforderlich, weil ja auch in jeder denkbaren historischen Wirklichkeit widerstreitende Wirkungsfaktoren, oft auf „dialektische“ Weise, miteinander um Vorherrschaft konkurrieren. Wenn es denn überhaupt eine Gemeinsamkeit zwischen den von mir im Folgenden als wirklich und möglich dargestellten, nach meiner Einschätzung über das heutige „deutsche System“ hinausweisenden Entwicklungen und Tendenzen gibt, so bestünde diese darin, dass die Institutionen, die die für Nachkriegsdeutschland charakteristische sozial ausgeglichene und ausgleichende Kooperation zwischen Arbeit und Kapital getragen haben und tragen, heute zunehmend weniger universell und universalistisch - alsonach innen heterogener und nachaußen wenigerflächendeckend - geworden sind und weiter werden, mit Konsequenzen für ihre Struktur und Stabilität, und dass ihr Funktionieren immer weniger an Politik, und damit an ein politisch formuliertes öffentliches Interesse, und immer mehr an den Markt gebunden ist und sein wird.

1. Zunächst mehren sich die Anzeichen, dass die Kontinuität des deutschen Systems der industriellen Beziehungen in den letzten Jahren zu einem guten Teil von dessen fortdauernder Subventionierung durch eine flankierende staatliche Sozial- und Arbeitsmarktpolitik abhing. Erforderlich ist diese zur Absorption der sozialen Kosten eines Lohnfindungssystems, das nur eine vergleichsweise geringe Lohndifferenzierung zwischen Unternehmen und Sektoren zuläßt und damit vor allem den Aufbau neuer Beschäftigung im Dienstleistungssektor behindert (Streeck 2001b). Überwogen in der Vergangenheit die produktivitätsfördernden Wirkungen des egalitären deutschen Lohnregimes - die unter anderem auf dem Weg über die von ihm motivierten Qualifizierungsanstrengungen der Unternehmen zustande kamen - so scheinen heute bei veränderten organisatorischen und wirtschaftlichen Rahmenbedingungen beschäftigungsverhindernde Effekte die Überhand gewonnen zu haben. Die traditionelle Prämisse der deutschen Lohn- und Arbeitsmarktpolitik, dass es besser sei, für einen notfalls auch längeren Übergangszeitraum Arbeitslosigkeit zu finanzieren, als ,schlechte“ Beschäftigung, höhere Ungleichheit und Flexibilisierung der Arbeitsmärkte hinzunehmen, hat sich in der Wirklichkeit eines Jahrzehnte andauernden Überhangs des Angebots am Arbeitsmarkt über eine institutionell restringierte Nachfrage zu einer etablierten Politik der Stillegung von überschüssig gemachter Arbeitskraft verfestigt, die zu wachsenden fiskalischen Kosten den Druck des Angebotsüberhangs auf den Arbeitsmarkt und seine Institutionen künstlich vermindert. Resultat ist ein Beschäftigungssystem, das sich im internationalen Vergleich zugleich durch niedrige Ungleichheit und hohe Inaktivität auszeichnet und, wie sein hoher und wachsender Subventionsbedarf ausweist, nicht mehr produktiv, sondern längst wirtschaftlich parasitär ist.

Wie lange das deutsche System der industriellen Beziehungen seine Kontinuität noch wird verteidigen können, würde demnach davon abhängen, wie lange seine Nutznießer im beschäftigten und seine ruhig gestellten Klienten im unbeschäftigten Teil der Gesellschaft den Staat dazu bewegen können, auf dem Weg über eine öffentliche Subventionierung der Sozialkassen einen Teil der Rechnung zu bezahlen. Die Aussichten hierfür wären, angesichts der Dominanz der organisierten Interessen der Arbeitnehmer und Arbeitgeber des industriellen Sektors im politischen System, grundsätzlich nicht schlecht, gäbe es nicht die gebiete- 
rische Forderung der internationalen Finanzmärkte und des europäischen Stabilitätspaktes nach Haushaltskonsolidierung, dem sich auf absehbare Zeit kein Finanzminister wird entziehen können. Allerdings verfügen die deutschen Sozialpartner über die Möglichkeit, sich eine Verlängerung der Gnadenfrist zu erkaufen, indem sie allfällige Kürzungen der Bundeszuschüsse zu den Kassen der Rentenversicherungsträger und der Bundesanstalt für Arbeit durch höhere Sozialbeiträge oder andere, neuartige Belastungen der aktiv Beschäftigten ausgleichen; eben dies war denn auch das unterliegende Thema der Auseinandersetzungen um die „Rente mit 60“, die Altersteilzeit und die „Rentenreform“.

2. Freilich vergrößert ein solches Vorgehen die ohnehin große Gefahr, dass die ,typisch deutsche" Privilegierung von qualifizierter und (annähernd gleich) hoch bezahlter Beschäftigung sowie die dafür notwendige sozialverträgliche Stillegung von überflüssig gemachter Arbeit zur Ursache von sozialer Exklusion werden. Der wirtschaftliche Mechanismus, der diesen Zusammenhang herstellt, sind die in Deutschland selbst im kontinentaleuropäischen Vergleich besonders hohen Lohnnebenkosten (Arbeitsgruppe Benchmarking 2000). Gewerkschaftliche Beschwörungen, denen zufolge die Arbeitskosten keinen Einfluss auf das Beschäftigungsniveau haben, verraten nur, dass der Sachverhalt als solcher seinen Urhebern beunruhigend gegenwärtig ist. Daran, dass in Deutschland die Kosten der Stillegung von Arbeitskraft, soweit sie aus Sozialbeiträgen bestritten werden, längst ein $\mathrm{Maß}$ erreicht haben, das die Nachfrage nach Arbeit weiter verringert und zusätzliche Stillegungsmaßnahmen erforderlich macht, die wiederum zu neuen Kostensteigerungen führen, können sie nichts ändern. Damit aber tritt neben die institutionell geförderte und geforderte relativ hohe Gleichheit der Bedingungen innerhalb des Beschäftigungssystems eine dauerhafte kategorische Ungleichheit zwischen denen, die ihm angehören, und der wachsenden Anzahl derer, die zu ihm keinen Zugang finden.

Unvermeidliche Folge ist die Verwandlung des deutschen Systems sozial gesicherter abhängiger Beschäftigung, das sich in der Nachkriegsperiode mit dem Versprechen politisch legitimieren konnte, die gesamte Arbeit der Gesellschaft zu grundsätzlich gleichen Bedingungen zu organisieren, in ein sozial exklusives partikulares und partikularistisches System, das nicht mehr ist und zu sein beanspruchen kann als Teil eines gesamtgesellschaftlichen Dualismus. Sein von ihm teilweise selbst erzeugtes Gegen-Teil ist das wachsende Zweit- bzw. Ersatzbeschäftigungssystem der 630-Mark-Jobs sowie die mittlerweile auf fünfzehn Prozent des Sozialprodukts angewachsene Schattenökonomie - eine Sonderwirtschaftszone, in der sich diejenigen legal, halblegal und illegal betätigen können, die wegen der hohen Kosten der sozialen Sicherung der Beschäftigten des primären Sektors weder zu diesem noch zu den Vorteilen jener Sicherungssysteme Zutritt finden, für die sie direkt nichts, indirekt aber umso mehr bezahlen. Politische Siege der Gewerkschaften im Kampf um gleichbleibende oder steigende Sozialbeiträge, die gebraucht werden, damit die Frühverrentung der Gewerkschaftsmitglieder und das durchschnittliche Renteneintrittsalter von unter 60 Jahren trotz sinkender Staatszuschüsse weiterhin finanziert werden können, sind dann zugleich Niederlagen, wenn nicht der aktiven Beitragszahler insgesamt, so doch jedenfalls der im informellen Sektor und vor allem der überhaupt nicht Beschäftigten. Je mehr dies so ist und auch wahrgenommen wird, desto schwerer muss es werden, anderen als den heutigen und in der unmittelbaren Zukunft neu zu kreierenden Frührentnern die subventionierte Stilllegung von Arbeitskraft als soziale Errungenschaft plausibel zu machen - und auf desto schwächeren Füßen müssen die Legitimität des ,deutschen Systems“ und insbesondere der für die 
politische Durchsetzungsfähigkeit der Gewerkschaften langfristig essentielle Anspruch stehen, nicht nur die eigenen Mitglieder, sondern alle Beschäftigten zu vertreten.

3. Weitere Indizien für eine in Gang befindliche partikularistisch-sektorale Einkapselung des deutschen Systems der industriellen Beziehungen liefern die nun schon sehr weit fortgeschrittene Konzentration der Mitbestimmung und des Flächentarifs auf den, unvermeidlich schrumpfenden, industriellen Sektor und auf traditionelle Großunternehmen, sowie die gleichzeitig zu beobachtende Konzentration der Gewerkschaftsmitgliedschaft auf die Generation derjenigen, die ihre politische und organisatorische Sozialisation in den roaring seventies erfahren haben. Gewerkschaften hatten es im Dienstleistungssektor, bei kleinen und neuen Unternehmen sowie bei jungen Menschen immer und überall schwer. Das Organisationsdefizit der deutschen Gewerkschaften in diesen Bereichen dauert heute aber schon so lange an und scheint ein so großes Ausmaß angenommen zu haben, dass sich der Eindruck aufdrängt, dass es sich bei ihm um mehr handelt als das übliche zeitliche Zurückhängen der organisatorischen hinter der wirtschaftlichen, strukturellen und demographischen Entwicklung. Vor allem in der ,neuen Ökonomie“ und den an Zahl und Bedeutung wachsenden Klein- und Mittelunternehmen sowie in der jüngeren Generation ist heute nicht mehr auszuschließen, dass die Präsenz der Gewerkschaften unaufholbar unter jene kritische Masse gesunken ist, die erforderlich wäre, damit ein selbsttragender Organisierungsprozess auch nur eine Chance hätte, wieder in Gang zu kommen.

Für den politischen Status und den zukünftigen Entwicklungspfad des deutschen Systems der industriellen Beziehungen müsste dies schwerwiegende Folgen haben. Auch bei ansonsten gleichbleibender Binnenstruktur und Funktionsweise käme eine partikularisierende Einkapselung in einen Teilbereich von Wirtschaft und Gesellschaft einem einschneidenden Gestaltwandel gleich. War das deutsche System der Arbeitsbeziehungen als ein national standardisiertes, alle industrial citizens gleichermaßen einschließendes Regime ursprünglich nicht zuletzt politisch begründet, so wäre seine sektorale Partikularisierung der Beginn einer Ausdifferenzierung unterschiedlicher, jeweils auf Teilbereiche von Wirtschaft und Gesellschaft beschränkter Beschäftigungssysteme, die wie in den USA der union sector und der non-union sector durchaus auch miteinander in Wettbewerb treten könnten. Unvermeidliche Folge wäre eine Unterminierung gewerkschaftlicher oder überhaupt kollektiver Vertretung überall dort, wo diese nicht an produktionstechnische oder wirtschaftliche Bedingungen anschließen kann, die sie auch aus Sicht der Arbeitgeber zu einem effizienten governanceArrangement machen.

Alternativ bzw. für eine (möglicherweise auch lange) Übergangszeit - nämlich bis zur endgültigen Verrentung der heutigen Gewerkschaftsmitglieder und -funktionäre oder zum erfolgreich abgeschlossenen Umbau der ,alten“ in eine „neue“ Ökonomie - bliebe als hauptsächliche Rechtfertigung der Existenz von Gewerkschaften, Tarifverträgen und Mitbestimmung nur noch, dass es sie eben in der Vergangenheit gegeben hat und es bis auf weiteres rational erscheint, sie zur Vermeidung von überflüssigen Streitereien ,,pfadabhängig“ weiterbestehen zu lassen, solange sie keinen Ärger machen - eine Erklärung, deren defensiver Charakter sie jedenfalls zur Werbung neuer Mitglieder durchaus ungeeignet macht ${ }^{2}$. In

2 Eine mögliche gewerkschaftliche, Revitalisierung“ließe sich dann wohl nur noch durch neue Organisationen vorstellen, etwa wie ansatzweise in den USA, der heute nicht repräsentierten, oft ausländischen Beschäftigten am unterenEnde des Dienstleistungssektors. Wenn solche Organisationen allerdings in Deutschland überhaupt jemals in Erscheinung treten sollten, stünde zu erwarten, dass sie sich gegen erhebliche Widerstände der bestehenden Gewerkschaften durchsetzen müßten. 
beiden Fällen - Einkapselung oder langsames Verschwinden - verlören die Gewerkschaften und das Regime sozial gesicherter Lohnarbeit, das sie nach dem Zweiten Weltkrieg geprägt haben, jeden Anspruch auf privilegierten Zugang zur Politik, einschließlich aller noch so sozialdemokratischen Parteien, aus deren Perspektive das Interesse des „organisierten Sektors“ dann immer mehr zu einem gegen andere Interessen abzuwägenden und jedenfalls zutiefst hegemonieunfähigen Spezialinteresse werden müßte ${ }^{3}$.

4. Auch die offenbar so erfolgreiche Anpassung der Mitbestimmung an die neuen Produktund Kapitalmärkte muss nicht unbedingt ausschließlich im Sinne institutioneller Kontinuität gedeutet werden. Die zu beobachtende Differenzierung der Mitbestimmungspraxis zwischen Unternehmen und Betrieben und die Herausbildung maßgeschneiderter, auf die jeweiligen wirtschaftlichen und technologischen Bedingungen abgestimmter Mitbestimmungsregime auf vertraglicher und quasi-vertraglicher Basis ist bekanntlich von der Kommission Mitbestimmung als institutioneller Reifungsprozess sowie als graduelle, die fundamentals des Systems nicht berührende Verbetrieblichung der Mitbestimmung dargestellt worden; sie ließe sich freilich ebenso auch als Folge eines allmählichen Herauswachsens der Mitbestimmung aus der Reichweite staatlicher Gestaltungsmacht und insoweit als Ausdruck einer faktischen Privatisierung der von ihr garantierten industriellen Bürgerrechte beschreiben. Privatisiert wäre Mitbestimmung in dem Maße, wie sie in ihrer tatsächlichen Ausübung immer weniger politisch-rechtlich und immer mehr wirtschaftlich fundiert und in ihrer Gestalt mehr ,,von unten“ im Unternehmen bzw. „,von der Seite“ im Wettbewerb als „von oben“ durch einen gleiche Rechtsansprüche konstituierenden Nationalstaat geprägt wäre. Die Folge wäre, dass der Status des industrial citizen, statt wie ursprünglich durch eine politische Verfassung der Wirtschaft als Teil der Gesellschaft, durch die marktbestimmten Zweckmäßigkeiten und Notwendigkeiten eines unternehmerischen human resource management definiert würde, das sich der traditionellen Institution der Mitbestimmung als Vehikel zum Aufbau solidarischer Wettbewerbsgemeinschaften in kompetitiver gewordenen Märkten bedient, indem sie diese vermittels einer allmählichen Kontraktualisierung ihrer Geschäftsgrundlage an ihre jeweiligen einzelbetrieblichen Zwecke anpasst.

Eine solche Entwicklung passte zu den oben beschriebenen Tendenzen zu einer allmählichen Einkapselung dessen, was dann als ancien régime der deutschen industriellen Beziehungen zu bezeichnen wäre. In dem Maße, wie die Perspektive einer politischen Generalisierung des Rechts auf industrielle Bürgerrechte von der Notwendigkeit der Bedienung einzelwirtschaftlicher Zwänge in den Hintergrund gedrängt wird, kann Mitbestimmung als quasi-privatisiertes unternehmensspezifisches Beteiligungsregime zur Herausbildung von eigentumsähnlichen Beschäftigungsrechten führen, also von faktischenjob property rights eines privilegierten Teils der Arbeitnehmerschaft. Einkapselung und Privatisierung würden im Übrigen weiter zunehmen, wenn auf Unternehmensebene zu den gesetzlich und quasi-vertraglich ausgeübten Mitbestimmungsrechten tatsächliche Eigentumsrechte in Gestalt von Belegschaftsaktien kämen, die ja ihrem Wesen nach ohnehin allein an die sogenannte „Kernbelegschaft“" ausgegeben werden können. In Unternehmen mit verstreutem Aktienbesitz könnte

3 Von der schon eingetretenen Einkapselung des deutschen Systems insgesamt in den institutionellen Flickenteppich des integrierten Europa soll hier nicht die Rede sein, obwohl sie einer ähnlichen Logik folgen und ähnliche Konsequenzen haben dürfte: ein Export des deutschen Systems nach Europa ist nicht mehr zu erwarten, ebensowenig wie die Etablierung eines anderen einheitlichen europäischen Systems, mit der Folge, dass die deutschen Institutionen Wege zum Überleben innerhalb von Systemkonkurrenz finden oder sich mit derEndspiel-Perspektive ihres langsamen Absterbens befreunden müssen. 
auf diese Weise die (Kern-) Belegschaft als Ganze, mit aktiver Unterstützung der Geschäftsleitung und womöglich repräsentiert durch ihren Betriebsrat, zum größten individuellen Anteilseigner werden und damit in der governance des Unternehmen auf beiden Seiten zugleich vertreten sein. Innerhalb eines auf solche Weise sozial hoch integrierten Unternehmens wären dann die Grenzen zwischen Arbeit und Kapital verwischt, auch weil die (Kern-) Arbeit als Produktionsfaktor kaum weniger fix wäre als das Kapital und die Belegschaft und ihre Vertreter keinen Grund mehr hätten, sich mit den Interessen des Unternehmens weniger zu identifizieren als seine (anderen) Eigentümer.

5. Allerdings ließe sich vorstellen, dass auch eine noch so weitgehende privatistische Einkapselung der Mitbestimmung nicht mehr als nur ein Übergangsstadium wäre. Dies wäre dann der Fall, wenn es zuträfe, dass die Mitbestimmung als Institution ein Modell von abhängiger Beschäftigung als Normalfall voraussetzt, das gerade auch für die großen Unternehmen, in denen die Mitbestimmung besonders gut gefestigt ist, nicht mehr lange stilprägend bleiben muss. Vieles spricht dafür, dass Mitbestimmung nur in dem Maße zugleich relevante Arbeitnehmerinteressen befriedigen und für den Arbeitgeber mehr Nutzen als Kosten mit sich bringen kann, wie Beschäftigungsverhältnisse typischerweise lange dauern und betriebsspezifische, nur in dem betreffenden Unternehmen erwerb- und verwertbare Qualifikationen für dessen Produktionsprozess von hoher wirtschaftlicher Bedeutung sind. Beides war in Deutschland lange Zeit der Fall. Arbeitnehmer, die von vornherein nur kurzfristig beschäftigt sind oder sein wollen, und insbesondere Arbeitnehmer, die über transportable generelle Qualifikationen verfügen, die von vielen Arbeitgebern gleichermaßen nachgefragt werden, entwickeln schon heute kein großes Interesse an dem Unternehmen, für das sie gerade arbeiten. Sie müssen entweder sowieso bald gehen, oder sie können gehen, wenn es ihnen woanders besser gefällt. Wenn in Zukunft zwischenbetrieblich übertragbare Qualifikationen wichtiger und dementsprechend Beschäftigungsverhältnisse kürzer würden - ein Muster, das sich in den neunziger Jahren jedenfalls im Top-Management großer deutscher Unternehmen entgegen allen Erwartungen der ,, Varieties of Capitalism “-Literatur bereits durchgesetzt zu haben scheint - dann müssten innerhalb der Belegschaften jene unternehmensbezogenen Interessen in den Hintergrund treten, zu deren kooperativer Verarbeitung die Institutionen der Mitbestimmung so ausgezeichnet geeignet sind. Und wenn Unternehmen sich in Zukunft die von ihnen am meisten benötigten Qualifikationen über einen flexiblen externen Arbeitsmarkt beschaffen und hohe Fluktuation nicht mehr gleichbedeutend mit einem Verlust kurzfristig nicht ersetzbarer Qualifikationen wäre, verlören die Arbeitgeber jeden Anreiz, ihren Arbeitskräften zur Verhinderung ihres Abgangs Gelegenheiten zur Mitsprache einzuräumen.

Ebenso und ähnlich wie durch einen Wandel der Qualifikationsstrukturen, des Qualifikationsbedarfes und der Arbeitsmarktdynamik könnte die Mitbestimmung durch eine Veränderung der betrieblichen Organisationsformen und Anreizstrukturen, wie sie sich heute vielerorts abzeichnet, an Rückhalt verlieren. Mitbestimmung auf dem Weg über einen aus der betrieblichen Hierarchie ausdifferenzierten, ,politischen“ Willensbildungsstrang korrigiert eine Reihe von Dysfunktionen einer fordistisch-bürokratischen Ausgestaltung der Arbeitnehmerrolle, die dem Beschäftigten formal nicht mehr abverlangt als gleichmäßige und verantwortungslose Leistungsablieferung bei im Wesentlichen motivloser Befolgung ,von oben" ergehender Anordnungen. Insoweit als derartige Dysfunktionen nicht nur für den Arbeitnehmer, sondern auch für den Arbeitgeber problematisch sein können, ist Mitbestim- 
mung zwischen beiden grundsätzlich konsensfähig. Neue Organisationsformen, die die strikte Trennung von Konzeption und Exekution bzw. von „Management“ und „Produktion“ aufweichen, könnten dann also Mitbestimmung in ihrer heutigen Form unnötig werden lassen; sie würde für die Regelung von Problemen nur noch in jenem - schrumpfenden Bereich in Anspruch genommen, in dem die Arbeitsbeziehungen weiterhin traditionell hierarchisch geprägt sind. Überall sonst würde sie über die wachsende Bedeutung spezialisierten Fachwissens und die Delegation von Entscheidungskompetenzen nach unten direkt in den Arbeitsvollzug als solchen integriert und käme ohne einen spezialisierten ,ppolitischen“ Kanal der Interessenartikulation aus - wobei die freiwillige Einbringung destacit knowledge der Beschäftigten unter anderem durch neue Entlohnungssysteme gesichert wäre, die die Interessen von Arbeitnehmer und Unternehmen mehr als bisher zur Deckung bringen. Insbesondere dort, wo der klassischeArbeitsvertragsnehmer de jure oder de facto durch einen Werkvertragsnehmer ersetzt wird, der Ausführung und Kosten seiner Tätigkeit selbst kontrolliert und einen Teil seiner Entlohnung als Residualeinkommen erhält, muss das Interesse an Mitbestimmung als Institution zum Ausgleich der Dysfunktionen einer hierarchischen Arbeitsorganisation auf beiden Seiten abnehmen.

Eine solche Vorhersage müsste mit dem Befund eines insgesamt unbestrittenen Fortbestands des deutschen Mitbestimmungsregimes nicht unvereinbar sein. Mitbestimmung durch spezialisierte Mitbestimmungsorgane könnte auch inmitten eines allgemeinen Übergangs zu einer postfordistischen Arbeits- und Unternehmensorganisation noch lange weitergehen; sie könnte dazu genutzt werden, den Übergang zu einer grundlegend neuen Organisation von Arbeit und Beschäftigung sozialfriedlich zu bewältigen, oder sie könnte zur Domäne der klassischen Arbeitnehmer in langfristigen Beschäftigungsverhältnissen werden, deren Leistung weiterhin nach ihrer aufgewendeten Arbeit statt nach deren Resultat bewertet würde. In der neuen Arbeitswelt freilich wäre die verbleibende Klientel der Mitbestimmung gerade keine privilegierte Kernbelegschaft - eine solche gäbe es dort nicht -, sondern sie bestünde aus den Immobilen und schlechter Ausgebildeten und somit besonders Schutzbedürftigen. Überflüssig würde die Mitbestimmung dadurch nicht. Aber als Schutzzone derjenigen, die nicht zu den „Leistungsträgern“ gehören, wäre Mitbestimmung immer mehr ein Kostenfaktor; sie verlöre die Unterstützung derjenigen, von deren Leistungsbeitrag das Unternehmen abhängt, und das Prestige, das aus solcher Unterstützung fließt; und sie könnte immer weniger beanspruchen, die Interessen der gesamten Belegschaft mit denen des Unternehmens als ganzem in Einklang zu bringen. Auch hier wäre damit zu rechnen, dass eine graduelle Schrumpfung der Mitbestimmung zu einem spezialisierten Regime zur Regulierung der Arbeitsverhältnisse einer immer kleiner werdenden Gruppe irgendwann in einen qualitativen Wandel umschlägt.

6. Schließlich wäre daran zu erinnern, dass die Mitbestimmung ebenso wie andere Elemente des deutschen Systems der industriellen Beziehungen immer auch zur Bindung wirtschaftlichen Handelns an ein öffentliches Interesse diente oder doch dienen sollte. Der mitbestimmungspolitische Durchgriff des Gesetzgebers in die innere Organisation, und das heißt: die Privatsphäre, des Großunternehmens öffnete diese für die Einwirkung unternehmensexterner, umfassendere Interessen repräsentierender Regulierungsinstanzen - etwa über die gesetzlich vorgeschriebene Präsenz externer Gewerkschaftsvertreter in den Aufsichtsräten mitbestimmter Unternehmen. Welche praktische Bedeutung gerade dieser Mechanismus voncorporate accountability heute, nach dem restlosen Verschwinden der gewerkschaftlichen Planungshoffnungen der Nachkriegszeit, noch hat, kann hier 
offengelassen werden. Aber die marktgetriebene Privatisierung der Grundlagen der Mitbestimmung in der Praxis und das tendenzielle Schrumpfen des formellen Systems der industriellen Beziehungen auf ein eingekapseltes Partialregime können jedenfalls als Zeichen für einen Rückgang der politischen Gestaltungskraft eines Nationalstaats angesehen werden, dem es immer weniger gelingt, die Binnensphäre und das Verhalten großer Unternehmen so zu regeln, dass sie sozialer Integration über Unternehmensgrenzen hinweg und einer egalitären Verteilung von industriellen Bürgerrechten in der Gesellschaft förderlich sind. Parallel dazu mag man sich daran erinnern, dass auch die Besteuerbarkeit der Großunternehmen zurückgeht, während ihre Ansprüche an die öffentlich zu erstellende physische und soziale Infrastruktur, einschließlich des sozialpolitisch zu sichernden sozialen Friedens, ständig steigen. Ebenfalls steigen die Kosten, die die Unternehmen auf die Gesellschaft überwälzen, etwa in Gestalt der zur Routine gewordenen Nutzung der Frühverrentung und der sogenannten ,,aktiven“ Arbeitsmarktpolitik zum Aufbau „olympiareifer Mannschaften“ und zur friedlichen Bewältigung des Strukturwandels innerhalb des Unternehmens.

Wie die Nationalstaaten in einer globalisierten Ökonomie jemals wieder in die Lage kommen sollen, ihre mächtigen corporate citizens zu so etwas wie Verantwortung zu ziehen, ist eine Frage, die hier nicht einmal annähernd behandelt werden kann. Sicher ist aber, dass heute wohl niemand auf die Idee käme, dass dies auf dem Weg über eine Ausweitung der betrieblichen Mitbestimmung oder der Mitbestimmung auf Unternehmensebene geschehen könnte. Auch insoweit hat die Politik heute das Interesse an Mitbestimmung - als eines Mechanismus zum Transport öffentlicher Interessen in den Arkanbereich des Großunternehmens - fast gänzlich verloren. Über neue Instrumente umweltpolitischer Regulierung oder über eine Neujustierung des Verhältnisses von betrieblicher Weiterbildung und öffentlichem Bildungssystem zur Verbesserung der employability der Beschäftigten wird viel nachgedacht. Aber an Mitbestimmung der Arbeitnehmer als Vollstrecker anderer als partikularer Interessen der beteiligten Arbeitnehmer selber glaubt niemand mehr. Mit dem Wandel der Legitimitationsbasis der Mitbestimmung - und insbesondere mit dem Verlust verallgemeinerungsfähiger Legitimitätsansprüche und einer weithin wahrgenommenen oder doch sich abzeichnenden Reduzierung von Gewerkschaften und Mitbestimmungsorganen auf „korporatistische" Interessenvertretung in italienischen oder französischen Sinn (in dem Korporatismus traditionell als Synonym für Betriebs- und Gruppenegoismus gebraucht wird) verändert sich die Institution selber und vor allem die Bedingungen ihrer Stabilität und Evolution.

\section{Literatur}

Arbeitsgruppe Benchmarking (2000): Möglichkeiten zur Verbesserung der Beschäftigungschancen gering qualifizierter Arbeitnehmer. Bericht der Wissenschaftlergruppe der Arbeitsgruppe Benchmarking des Bündnisses für Arbeit, Ausbildung und Wettbewerbsfähigkeit. Presse- und Informationsamt der Bundesregierung, Berlin

Kommission Mitbestimmung (1998): Mitbestimmung und neue Unternehmenskulturen: Bilanz und Perspektiven. Bericht der Kommission Mitbestimmung. Gütersloh

Soskice, David (1999): Divergent Production Regimes: Coordinated and Uncoordinated Market Economies in the 1980s and 1990s; in: Herbert Kitschelt u.a. (Hg.): Continuity and Change in Contemporary Capitalism. Cambridge, 101-134 
Schroeder, Wolfgang, Burkard Ruppert (1996): Austritte aus Arbeitgeberverbänden: Eine Gefahr für das deutsche Modell? Marburg

Streeck, Wolfgang (2001a): High Equality, Low Activity: The Contribution of the Social Welfare System to the Stability of the German Collective Bargaining Regime. Review Symposium on Harry C. Katz and Owen Darbishire, Converging Divergences: Worldwide Changes in Employment Systems; in: Industrial and Labor Relations Review, Bd. 54, Nr. 3, 698-706

Streeck, Wolfgang (2001b): Tarifautonomie und Politik: Von der Konzertierten Aktion zum Bündnis für Arbeit; in: Gesamtverband der metallindustriellen Arbeitgeberverbände (Hg.): Die deutschen Arbeitsbeziehungen am Anfang des 20. Jahrhunderts. Wissenschaftliches Kolloquium aus Anlass des Ausscheidens von Dr. Werner Stumpfe als Präsident von Gesamtmetall, Köln, 76-102

Swenson, Peter (1997): Arranged Alliance: Business Interests in the New Deal; in: Politics and Society, Bd. 25, Nr. 1, 66-116

Thelen, Kathleen (1999): Why Employers Cannot Bring Themselves to Abandon the German Model; in: Torben Iversen, Jonas Pontusson, David Soskice (Hg.): Unions, Employers and Central Banks: Wage Bargaining and Macro-Economic Regimes in an Integrating Europe. New York

Traxler, Franz (1996): Collective Bargaining and Industrial Change: A Case of Disorganization? A Comparative Analysis of Eighteen OECD Countries; in: European Sociological Review, Bd. 12, Nr. 3, 271-287

Anschrift des Verfassers:

Prof. Dr. Wolfgang Streeck

Max-Planck-Institut für Gesellschaftsforschung Köln

Paulstraße 3

D-50676 Köln

\section{Schlagwörter: Arbeitsbeziehungen, Gewerkschaften, Globalisierung, Mitbestimmung, Strukturwandel}

